\title{
Circulating soluble fms-like tyrosine kinase-1, soluble endoglin and placental growth factor during pregnancy in normotensive women in KwaZulu-Natal, South Africa
}

\author{
Muhammed Ogunlola ${ }^{1}$, Poovendhree Reddy ${ }^{1}$, Maureen N. Sibiya ${ }^{2}$, Laura O’Connor ${ }^{3}$, \\ Dorinda Borg ${ }^{3}$, Firoza Haffejee ${ }^{5}$, Shanaz Ghuman ${ }^{1}$, Thembelihle Ngxongo ${ }^{4}$, Nalini Govender ${ }^{5}$
}

1. Department of Community Health Studies, Faculty of Health Sciences, Durban University of Technology.

2. Faculty of Health Sciences, Durban University of Technology, Durban, South Africa.

3. Department of Chiropractic and Somatology, Faculty of Health Sciences, Durban University of Technology, SA.

4. Department of Nursing, Faculty of Health Sciences, Durban University of Technology, SA.

5. Department of Basic Medical Sciences, Durban University of Technology, Durban, South Africa.

\section{Emails:}

muhaogunlola@yahoo.com; Contact number: 0742249999- PoovieR@dut.ac.za; Contact number: 031 373-2808 nokuthulas@dut.ac.za; Contact number:031373-2704-lauraw@dut.ac.za; Contact number:031 373-2923-dorindab@ dut.ac.za; Contact number: 031 373-2390-firozah@dut.ac.za; Contact number: 031 373-2395-shanazg@dut.ac.za; Contact number:031373-2807-thembelihlen@dut.ac.za; Contact number:031373-2609-nalinip@dut.ac.za; Contact number: 031 373-2796

\section{Abstract}

Background: Based on the increased pre-eclampsia and HIV antenatal incidence in South Africa, we determined the angiogenic profiles due to its mechanistic link in preeclampsia development, throughout uncomplicated pregnancies in HIV positive and negative women.

Objective: To determine the angiogenic profiles throughout uncomplicated pregnancies in HIV positive and HIV negative women. We explored possible correlations between angiogenic serum levels and selected maternal characteristics (HIV status, gestational age, maternal factors, and pregnancy outcomes).

Method: This study was conducted at a primary health care facility in Durban, South Africa. Forty-six pregnant women aged 18-45 years, were enrolled at 10-20, 22-30 and 32-38 weeks' gestation, respectively through convenient sampling. Serum samples were collected and quantitatively evaluated using ELISAs. Clinical and epidemiological data were analysed using STATA (version 14). A probability level of $\mathrm{p}<0.05$ was considered statistically significant.

Results: Of those enrolled, $28.3 \%$ were nulliparous, $82 \%$ were HIV positive and none developed pre-eclampsia. Systolic and diastolic blood pressure increased slightly throughout pregnancy. Fluctuating angiogenic and anti-angiogenic levels were demonstrated during pregnancy.

Conclusion: This study contributes to the current angiogenic knowledge in normotensive pregnancies, and may assist as a reference range against which these factors may be compared in HIV complicated pregnancies.

Keywords: sFlt-1, PlGF, sEng, pregnancy, HIV.

DOI: https://dx.doi.org/10.4314/ahs.v19i2.4

Cite as: Ogunlola M, Reddy P, Sibiya MN, O'Connor L, Borg D, Haffejee F, Gbuman S, Ngxongo T, Govender N. Circulating soluble fmslike tyrosine kinase-1, soluble endoglin and placental growth factor during pregnancy in normotensive women in KwaZulu-Natal, South Africa. Afri Health Sci.2019;19(2): 1821-1832. bttps:/ / dx.doi.org/10.4314/abs.v19i2.4

\section{Corresponding author: \\ Nalini Govender, \\ Department of Basic Medical Sciences \\ Faculty of Health Sciences, Durban University \\ of Technology Durban, South Africa, 4001 \\ Email: nalinip@dut.ac.za}

\section{Introduction}

Despite the significant decrease in maternal morbidity and mortality worldwide, the WHO 2015 estimates highlight that approximately 303, 000 maternal deaths will occur by 2015 , with a maternal mortality rate of 216 per 100,000 live births during pregnancy, at delivery or postnatally, most cases occurring in low- and middle-income coun- 
tries $^{1}$. Suboptimal antenatal care in South Africa is linked with poorer uptake of Human Immunodeficiency Virus (HIV) testing and adverse maternal and birth outcomes ${ }^{2,3}$. In developing and resource constrained countries such as South Africa, HIV is responsible for an estimated $40 \%$ of all maternal deaths ${ }^{4}$. The antenatal HIV prevalence of approximately $40 \%$ is the highest in KwaZulu-Natal, South Africa ${ }^{5}$. To date, the most effective intervention used to prevent mother-to-child-transmission is the use of highly active antiretroviral treatment (HAART) ${ }^{4}$, which alters maternal immunological responses thus improving maternal and perinatal outcomes ${ }^{4,6}$. Despite the obvious benefits of HAART in pregnant women, the rates of adverse birth outcomes is still reportedly higher in HIV positive women on HAART than HIV-uninfected women ${ }^{6,7}$. Moreover, earlier studies have shown inconsistencies in the angiogenic expression in women on anti retrovirals in comparison to HIV uninfected women ${ }^{8,9}$, whilst a more recent South African study suggest that HIV possibly jeopardizes vascular perfusion during pregnancy, particularly placental development ${ }^{10}$.

Fetal development relies on adequate placental growth which is dependent on branching and non branching angiogenesis ${ }^{11,12}$. Branching angiogenesis occurs during the first 24 weeks of pregnancy, under relative hypoxic conditons, in contrast to third trimester non branching angiogenesis ${ }^{12}$. Placental trophoblasts secrete angiogenic placental growth factor (PlGF) and vascular endothelial growth factor (VEGF) into the maternal vasculature, supporting endothelial proliferation and survival, and arterial remodeling ${ }^{13,14}$. The bioavailability of both VEGF and PlGF is controlled by the anti-angiogenic factor soluble fms-like tyrosine kinase-1 (sFlt-1), which is a splice variant of the VEGF receptor ${ }^{15,16}$. Likewise, increased circulating levels of soluble endoglin (sEng) was also demonstrated and implicated in PE development ${ }^{17}$. Moreover, this placental derived protein competes with endoglin to bind to circulating transforming growth factor beta (TGF- $\beta$ ), thus weakening its signaling ${ }^{17}$. Walshe et al. ${ }^{18}$ suggests that the impaired signaling of VEGF and/or TGF- $\beta$ pathways may contribute to the endothelial dysnfunction that characterizes PE development. This disrupted angiogenic expression is linked with fetal growth restriction ${ }^{19}$, preterm delivery ${ }^{20}$ as well as hypertensive disorders of pregnancy (HDP), including preeclampsia $(\mathrm{PE})^{21}$, coronary artery disease ${ }^{22}$, heart failure and maternal vascular malperfusion10. It is possible that the altered placental perfusion contributes systemic alterations in the circulating expression of angiogenic factors ${ }^{23}$.

Pregnancy induced hypertensive disorders like PE are believed to occur in response to an immune-induced placental alteration evoked possibly by HIV and HAART mediated immune disturbances ${ }^{10}$. PE is characterized by increased circulating sFlt-1 levels, which inhibit angiogenic signaling and subsequently induce endothelial dysfunction $^{24}$. Placental delivery is no longer an option for PE resolution since epidemiological evidence confirms the onset of various long-term cardiovascular disorders, long after the dissolution of preeclampsia symptoms ${ }^{21,25}$. Despite the improved understanding of PE, delayed childbearing combined with maternal body mass index (BMI), maternal age, and parity, may be correlated with angiogenic balance even in uncomplicated pregnancies ${ }^{26,27}$.

Earlier studies demonstrates the clinical role of maternal PlGF and sFlt-1 levels as valuable predictors of preeclampsia development and its associated consequences in both mother and fetus ${ }^{28,29}$, thereby supporting their increased use in both maternal and fetal investigations because of its link with placental development $t^{30}$. Others have also recommended that evaluating maternal concentrations of both sFlt-1 and sEng may be helpful in the prognosis of pregnancy complications such as preeclampsia and intrauterine growth restriction ${ }^{19,31,32}$. Whilst we together with several others, have evaluated the angiogenic profiles in normal pregnancies as a means of comparing them with complicated pregnancies, the intention of such data was not intended at instituting reference values $^{28,33,34}$.

We therefore aimed to determined the angiogenic and anti-angiogenic profiles through three gestational periods from uncomplicated pregnancies in HIV positive (HIV + ve) and HIV negative (HIV -ve) women. We further explored possible correlations between serum levels of these analytes and selected maternal characteristics (HIV status, gestational age, maternal factors, and pregnancy outcomes). Since there was limited South African data, 
we intended to contribute to the current knowledge on serum levels of sFlt-1, PlGF and soluble endoglin (sEng).

\section{Methods}

This was a prospective study which was nested within a birth cohort study. It was conducted at a primary health care (PHC) facility in Durban, South Africa between 2015 and 2016. Antenatal care in South Africa is usually initiated before 20 weeks of gestation as prescribed by the National Department of Health ${ }^{35}$. Only participants who presented for their first antenatal visit, prior to 20 weeks gestation and who consented to blood collection at the 3 gestational periods were included in the study, which resulted in a final convenient sample number of 46. Participants could not be followed until delivery, since PHC facilities feed into various public health hospitals, resulting in logistical challenges in retaining all participants until delivery. Adolescent pregnancies ( $<18$ yrs), women presenting with chronic diabetes, chronic hypertension, gestational diabetes, connective tissue disorder, cardiac disease, sickle cell disease, anti-phospholipid antibody syndrome, chorioamnionitis, unknown HIV status, and those unable to provide informed consent were excluded. Following institutional (Durban University o Technology Institutional Research EthicsCommittee, IREC 045/14) and department of health (HRKM 234/14) permission, written and informed consent was obtained from all participants recruited through convenient sampling. Blood samples were collected between 10-20 weeks, 22-30 weeks and 32-38 weeks via venipuncture in serum separator tubes from pregnant women aged $18-45$ years, every time blood pressure was measured. Clinical and demographic variables including maternal age, weight and blood pressure, BMI, parity, HIV status, maternal hemoglobin $(\mathrm{Hb})$ levels and birth weight, were derived from chart registers and administered epidemiological questionnaires respectively. HIV status was confirmed at the first antenatal visit and if HIV positive, were immediately placed on antiretroviral therapy for prevention of mother to child transmission. Blood samples were centrifuged at 3500 $\mathrm{rpm}, 4^{\circ} \mathrm{C}$ for $5 \mathrm{~min}$ and serum samples were aliquoted and stored at $-80^{\circ} \mathrm{C}$ until analysis. Serum concentrations of sFlt-1 (1:5), sEng (1:5) and PlGF (1:2) were measured in triplicate by ELISA according to the manufacturer's protocol (R\&D Systems, Minneapolis, MN).

\section{Statistical analysis}

All statistical analyses were conducted using STATA (version 14). Data were assessed for their distribution by the Shapiro-Wilk test. The homogeneity of variance between groups was assessed by Levene's test for equality of error variances. Descriptive statistics was utilized and outcome variables are presented as means and median (interquartile range) depending on data distribution. Parity was a dichotomous variable evaluated as nulliparous or multiparous. BMI was evaluated both as a continuous and categorical variable. BMI was dichotomized into $\leq 24.99$ $\mathrm{kg} / \mathrm{m}^{2}$ (normal) and $>25 \mathrm{~kg} / \mathrm{m}^{2}$ (overweight). The Friedman's ANOVA test was used to compare the levels of biomarkers stratified by parity, BMI and HIV status. The Spearmans rank correlation test was computed to assess the relationship between serum levels of PlGF, sFlt-1 and sEng and maternal factors. A probability level of $p<0.05$ was considered statistically significant.

\section{Results}

A total of 46 participants were followed at 10-20, 22-30 and 32-38 weeks' gestation, respectively. Demographic and clinical characteristics are presented in Table 1. 
Table 1: Clinical characteristics of maternal cohort with normotensive pregnancies $(\mathbf{n}=4$

\begin{tabular}{|c|c|}
\hline Maternal Characteristics & \\
\hline Maternal age at delivery (yrs) (Mean, SD) & $25.76(5.01)$ \\
\hline Smoking $(\mathrm{n}, \%)$ & $2(4.35)$ \\
\hline Alcohol use (n, \%) & $4(8.70)$ \\
\hline Height (cm)(mean, SD) & $1.59(0.57)$ \\
\hline \multicolumn{2}{|l|}{ Weight (kg) (median, IQR) } \\
\hline $10-20$ weeks & $62.60(60.00-71.00)$ \\
\hline 22-30 weeks & $68.15(63.00-74.30)$ \\
\hline 32-38 weeks & $72(66.40-80.00)$ \\
\hline \multicolumn{2}{|c|}{ Body Mass Index (BMI, kg/m²) (median, IQR) } \\
\hline $10-20$ weeks & $25.27(22.74-28.53)$ \\
\hline 22-30 weeks & $27.15(23.95-30.52)$ \\
\hline 32-38 weeks & $28.35(25.85-32.46)$ \\
\hline \multicolumn{2}{|l|}{ Blood pressure (mmHg)(mean, SD) } \\
\hline \multicolumn{2}{|l|}{ Systolic } \\
\hline $10-20$ weeks & $101.50(11.05)$ \\
\hline 22-30 weeks & $108(10.21)$ \\
\hline 32-38 weeks & $110(10.30)$ \\
\hline \multicolumn{2}{|l|}{ Diastolic } \\
\hline $10-20$ weeks & $60(8.12)$ \\
\hline 22-30 weeks & $68(9.22)$ \\
\hline 32-38 weeks & $70(14.32)$ \\
\hline \multicolumn{2}{|l|}{ Parity (n, \%) } \\
\hline Nulliparous & $13(28.26)$ \\
\hline Primiparous/multiparous & $33(71.74)$ \\
\hline \multicolumn{2}{|l|}{ HIV Status (n, \%) } \\
\hline Positive & $38(82.61)$ \\
\hline Negative & $8(17.39)$ \\
\hline Birth Weight $(\mathrm{kg})$ (mean, SD) & $3.01(0.40)$ \\
\hline
\end{tabular}

The mean maternal age at delivery was 25 years, whilst the mean birth weight was $3.01 \mathrm{~kg}$. Of the total sample, $28 \%$ were nulliparous and $82 \%$ were HIV positive. Both systolic and diastolic blood pressure increased slightly throughout the defined gestational intervals. Median circulating levels of PlGF, sFlt-1 and sEng throughout pregnancy are presented in Table 2. Both PlGF and sFlt-1 levels increased throughout pregnancy, while soluble endoglin dropped around 30 weeks' gestation and thereafter stabilized to its initial levels. In contrast, both the indices of vascular disturbance (sFlt-1/PlGF and sFlt-1+sEng/ PlGF), decreased considerably towards the end of $2^{\text {nd }}$ and $3^{\text {rd }}$ trimesters.

Table 2: Serum levels of PIGF, sFlt-1, sEng and antiangiogenic ratios sFIt-1/PIGF; (sFlt-1 + sEng)/PIGF (Median, IQR, $n=46$ )

\begin{tabular}{|c|c|c|c|c|c|}
\hline \multirow{2}{*}{$\begin{array}{c}\text { Gestational } \\
\text { period (wks) }\end{array}$} & \multirow{2}{*}{$\begin{array}{l}\text { Angiogenic factor } \\
\text { PIGF pg/mL }\end{array}$} & \multicolumn{2}{|c|}{ Antiangiogenic factor } & \multicolumn{2}{|c|}{ Antiangiogenic ratios } \\
\hline & & sFlt1 pg/mL & sEng $(\mathbf{n g} / \mathbf{m l})$ & sFlt1/PIGF & $\begin{array}{c}\text { (sFlt1 + } \\
\text { sEng)/PIGF }\end{array}$ \\
\hline $10-20 \mathrm{wks}$ & $301.52(201.71)$ & $1169.17(825.12)$ & $5.08(1.99)$ & $5.23(11.3)$ & $22.31(35.02)$ \\
\hline $22-30 \mathrm{wks}$ & $642.48(374.27)$ & $1244.17(936.42)$ & $3.94(1.65)$ & $2.20(2.24)$ & $8.20(7.42)$ \\
\hline $32-38$ wks & $713.53(534.28)$ & $1358.07(870.87)$ & $5.33(3.06)$ & $1.81(2.71)$ & $7.55(5.89)$ \\
\hline
\end{tabular}


Median serum levels of PlGF, sFlt-1 and sEng throughout pregnancy, stratified by parity and BMI are shown in Table 3. There were no statistically significant differences noted based on this stratification. Median PlGF levels in multiparous women increased progressively throughout pregnancy however, in nulliparous women, there was an increase in serum PIGF levels between 10-30 weeks with a reduction of $130 \mathrm{pg} / \mathrm{ml}$ at 32 weeks' gestation (Table 3). In contrast, sFlt-1 levels dropped between 22-30 weeks in nulliparous women compared to multiparous and thereafter increased. Likewise, a reduction in sEng levels at 22-30 weeks' gestation was noted amongst the nulliparous and multiparous women, which subsequently increased at 32 week's (Table 3). With regard to the effect of BMI on the angiogenic profiles, PlGF levels increased progressively throughout pregnancy, irrespective of BMI $<24.99$ or $>25.00 \mathrm{~kg} / \mathrm{m}^{2}$. The concentration of sFlt-1 increased amongst those who had a BMI $<24.99 \mathrm{~kg} /$ $\mathrm{m}^{2}$ in contrast to those $>25.00 \mathrm{~kg} / \mathrm{m}^{2}$. Levels of $\mathrm{sEng}$ however declined between 22-30 weeks and thereafter increased at 32 week's irrespective of BMI $<24.99$ or $>$ $25.00 \mathrm{~kg} / \mathrm{m}^{2}$ (Table 3). There were no statistically significant differences observed based on this stratification.

Table 3: Effect of parity and BMI on angiogenic and antiangiogenic factors stratified by gestational periods i normotensive pregnancies $(n=46$, median, $I Q R)$

\begin{tabular}{|c|c|c|c|c|}
\hline \multicolumn{3}{|c|}{ Parity } & \multicolumn{2}{|c|}{ BMI } \\
\hline Angiogenic Profiles & Nulliparous & Multiparous & $\leq 24.9$ & $\geq 25$ \\
\hline PIGF (10 -20 wks) & 244.08 ( 81.14-340.59) & $307.58(143.89-398.16)$ & $\begin{array}{l}316.28(161.10- \\
371.88)\end{array}$ & $\begin{array}{l}236.73(139.11- \\
326.25)\end{array}$ \\
\hline PIGF (22-30 wks) & $757.13(483.53-886.61)$ & $569.02(420.38-798.07)$ & $\begin{array}{l}\text { 752.81(486.53- } \\
862.19)\end{array}$ & $\begin{array}{l}480.85(350.63- \\
771.40)\end{array}$ \\
\hline PIGF (32-38 wks) & $627.04(444.79-1059.38)$ & $713.53(247.80-854.24)$ & $\begin{array}{l}808.05 \text { (483.86- } \\
956.55)\end{array}$ & $\begin{array}{l}483.86(200.58- \\
784.72)\end{array}$ \\
\hline sFIt-1 (10 -20 wks) & $1457.98(955.80-1671.09)$ & $956.87(582.75-1422.34)$ & $\begin{array}{l}1033.15(779.87- \\
1705.77)\end{array}$ & $\begin{array}{l}1335.08(625.08- \\
1457.98)\end{array}$ \\
\hline sFIt-1 (22 -30 wks) & $1244.14(939.25-1748.67)$ & $\begin{array}{l}1260.31(790.72- \\
1724.39)\end{array}$ & $\begin{array}{l}1384.33(882.82- \\
1778.57)\end{array}$ & $\begin{array}{l}1228.58(746.33- \\
1642.00)\end{array}$ \\
\hline sFIt-1 (32- 38 wks) & 1594.77 (971.57-1996.57) & $\begin{array}{l}1210.92(750.61- \\
1594.03)\end{array}$ & $\begin{array}{l}1427.05(1056.63- \\
1779.14)\end{array}$ & $\begin{array}{l}1093.91(606.50- \\
1594.77)\end{array}$ \\
\hline sEng (10 -20 wks) & $5.08(3.97-5.76)$ & $5.08(4.28-6.72)$ & $5.52(4.85-6.72)$ & $4.77(4.10-5.78)$ \\
\hline sEng (22 -30 wks) & $4.01(3.54-5.77)$ & $3.85(3.58-4.79)$ & $4.18(3.67-5.77)$ & $3.79(3.54-5.23)$ \\
\hline sEng (32 -38 wks) & $5.13(4.10-7.79)$ & $5.33(4.67-7.14)$ & $5.33(4.41-6.59)$ & $5.32(4.63-7.82)$ \\
\hline sFIt1/PIGF (10-20 wks) & $6.94(3.36-13.46)$ & $4.01(1.36-8.93)$ & $4.63(1.46-8.67)$ & $6.94(2.27-13.86)$ \\
\hline sFIt1/PIGF (22-30 wks) & $2(1.20-3.58)$ & $2.21(1.86-3.25)$ & $2.00(1.20-2.49)$ & $2.47(1.58-5.67)$ \\
\hline sFIt1/PIGF (32-38 wks) & $1.99(0.96-2.46)$ & $1.73(1.04-3.67)$ & $1.81(1.04-2.46)$ & $1.93(0.90-3.82)$ \\
\hline $\begin{array}{l}\text { (sFIt1+sEng)/PIGF (10- } \\
20 \text { wks) } \\
\text { (sFIt1+sEng)/PIGF (22- } \\
30 \text { wks) } \\
\text { (sFIt1+sEng)/PIGF (32- } \\
38 \text { wks) }\end{array}$ & $\begin{array}{l}7.27(4.34-8.15) \\
5.99(5.80-9.42)\end{array}$ & $22.22(11.97-55.03)$ & $19.09(11.18-44.98)$ & $\begin{array}{l}29.05(7.24- \\
73.13) \\
12.79(4.34- \\
23.16)\end{array}$ \\
\hline
\end{tabular}

Medians of the angiogenic profiles and selected clinical parameters, stratified by HIV status, are shown in Table 4. The PlGF levels in the HIV+ve group increased throughout the defined gestational intervals, in comparison to thosewho were HIV-ve. Whilst higher sFlt-1 levels was noted in the HIV+ve group during the 10-20 weeks gestational interval compared to to the HIV-ve group, a reduction in their expression was observed during the latter gestational intervals. In contrast, sEng levels remained somewhat stable, but declined mid-gestation and thereafter stabilized to their initial expression levels (Table 4).
The indices of vascular disturbance (sFlt-1/PlGF and sFlt-1+sEng/PlGF), decreased considerably towards the end of the $2^{\text {nd }}$ and $3^{\text {rd }}$ gestational intervals in both the $\mathrm{HIV}+$ ve and -ve groups. However, the sFlt-1+sEng/ $\mathrm{PlGF}$ ratio was much higher in the HIV+ve compared to the HIV-ve groups only in weeks 22-38. Additionally, the median hemoglobin levels was slightly higher in the HIV-ve group, whilst BMI and birth weight were almost similar (Table 4). Both the systolic and diastolic blood pressures were slightly higher in the HIV-ve groups throughout the defined gestational intervals compared to the HIV+ve groups. 
Table 4: Angiogenic and antiangiogenic factors in normotensive pregnancy stratified by HIV status $(n=46)$

\begin{tabular}{|c|c|c|}
\hline Clinical characteristics & HIV -ve & HIV +ve \\
\hline \multicolumn{3}{|l|}{ PIGF (pg/ml, median, range) } \\
\hline 10-20wks & $240.4(162.8)$ & $308.08(346)$ \\
\hline 22-30wks & $771.4(374.27)$ & $608.09(428.8)$ \\
\hline $32-38 w k s$ & $483.86(488.25)$ & $721.5(595.98)$ \\
\hline \multicolumn{3}{|l|}{ sFlt-1(pg/ml, median, range) } \\
\hline 10-20wks & $819.69(851.04)$ & 1246.43 (799.57) \\
\hline $22-30 w k s$ & $1335.78(981.01)$ & $1244.14(935.09)$ \\
\hline $32-38 w k s$ & $1663.97(505.7)$ & $1230.05(857.52)$ \\
\hline \multicolumn{3}{|l|}{ sEng (ng/ml, median, range) } \\
\hline 10-20wks & $5.02(5.64)$ & $5.08(1.75)$ \\
\hline 22-30wks & $3.88(1.97)$ & $4.01(1.69)$ \\
\hline $32-38 w k s$ & $5.67(2.37)$ & $5.32(3.38)$ \\
\hline \multicolumn{3}{|l|}{ sflt/PIGF (median, range) } \\
\hline 10-20wks & $4(3.58)$ & $5.37(11.3)$ \\
\hline $22-30 w k s$ & $1.58(2.81)$ & $2.21(1.39)$ \\
\hline $32-38 w k s$ & $2.69(2.55)$ & $1.81(1.5)$ \\
\hline \multicolumn{3}{|c|}{ sFlt-1+sEng/PIGF (median, range) } \\
\hline $10-20 w k s$ & $33.03(54.54)$ & $22.31(38.5)$ \\
\hline $22-30 w k s$ & $5.98(12.5)$ & $8.53(6.92)$ \\
\hline $32-38 w k s$ & $5.51(3.09)$ & $8.16(5.4)$ \\
\hline BMI $\left(\mathrm{kg} / \mathrm{m}^{2}\right.$, mean, $\left.\mathrm{SD}\right)$ & $29.26(3.16)$ & $29.83(7.32)$ \\
\hline Hemoglobin (g/dL, mean, SD) & $11.78(0.87)$ & $10.63(1.54)$ \\
\hline Birth weight (kg, mean, SD) & $3.16(0.35)$ & $2.98(0.41)$ \\
\hline \multicolumn{3}{|l|}{ Diastolic blood pressure $(\mathrm{mmHg})$} \\
\hline $10-20 \mathrm{wks}$ & $63(9)$ & $60(2)$ \\
\hline $22-30$ wks & $79(16)$ & $67(8)$ \\
\hline $32-38$ wks & $75(16)$ & $69(12)$ \\
\hline \multicolumn{3}{|l|}{ Systolic blood pressure $(\mathrm{mmHg})$} \\
\hline $10-20$ wks & $113(30)$ & $100(10)$ \\
\hline $22-30$ wks & $110(11)$ & $106(10)$ \\
\hline $32-38$ wks & $112(24)$ & $110(10)$ \\
\hline
\end{tabular}

Correlations between angiogenic profiles and selected clinical characteristics at the defined gestational intervals are shown in Table 5. Maternal age was positively correlated with both indices of vascular disturbance (sFlt-1/ PlGF and sFlt-1+sEng/PlGF) at 32-38 weeks' gestation $(\mathrm{p}<0.05)$. In addition, a significant but negative correlation was noted between sFlt-1 and those with a BMI $>25.00 \mathrm{~kg} / \mathrm{m}^{2}$ at $10-20$ weeks. Despite the minimal increase noted in both systolic and diastolic blood pressure throughout the defined gestational intervals, an inverse and significant correlation was observed between systolic blood pressure and PlGF and sFlt-1 at 32-38 weeks' gestation respectively. A statistically significant positive correlation $(r=0.50)$ was observed between diastolic blood pressure and the antiangiogenic ratio (sFlt-1/PlGF) at 32-38 weeks' gestation. A weak positive correlation was noted between hemaglobin and sFlt-1 $+\mathrm{sEng} / \mathrm{PlGF}$ ratio at 32-38 weeks' gestation. 
Table 5: Pearson's correlations between clinical characteristics and angiogenic/antiangiogenic factors in normotensive pregnancies $(n=46)$

\begin{tabular}{|c|c|c|c|c|c|}
\hline \multirow[t]{2}{*}{ Clinical factor } & \multirow{2}{*}{$\begin{array}{c}\text { Angiogenic } \\
\text { factor } \\
\qquad \text { PIGF }\end{array}$} & \multicolumn{2}{|c|}{ Antiangiogenic factors } & \multicolumn{2}{|c|}{ Antiangiogenic ratios } \\
\hline & & sFlt-1 (pg/ml) & sEng $(\mathbf{n g} / \mathbf{m l})$ & sFlt1/PIGF & sEng)/PIGF \\
\hline \multicolumn{6}{|c|}{ Maternal age (years) } \\
\hline $10-20 w k s$ & 0,08 & $-0,29$ & 0,1 & $-0,37$ & $-0,31$ \\
\hline $22-32 w k s$ & $-0,11$ & $-0,09$ & 0,02 & $-0,07$ & $-0,06$ \\
\hline $32-38 w k s$ & $-0,13$ & 0,13 & $-0,1$ & $0.61 *$ & $0.01 *$ \\
\hline \multicolumn{6}{|l|}{ BMI $\left(\mathrm{kg} / \mathrm{m}^{2}\right)$} \\
\hline $10-20 w k s$ & 0,09 & $-0,02$ & 0,41 & $-0,43$ & $-0,23$ \\
\hline $22-32 w k s$ & $-0,36$ & $-0,31$ & $-0,22$ & 0,19 & $-0,14$ \\
\hline $32-38 w k s$ & $-0,03$ & 0,19 & 0,41 & 0,85 & 0,6 \\
\hline \multicolumn{6}{|l|}{$>25.00$} \\
\hline $10-20 w k s$ & 0 & $-0.47 *$ & $-0,06$ & $-0,19$ & 0,04 \\
\hline $22-32 w k s$ & 0,08 & $-0,25$ & $-0,27$ & $-0,16$ & 0,19 \\
\hline $32-38 w k s$ & 0,04 & $-0,2$ & $-0,14$ & $-0,33$ & 0,06 \\
\hline \multicolumn{6}{|c|}{ Blood Pressure (mmHg) } \\
\hline \multicolumn{6}{|c|}{ Systolic } \\
\hline $10-20 w k s$ & 0,07 & 0,08 & 0,11 & $-0,1$ & $-0,09$ \\
\hline $22-32 w k s$ & 0,17 & $-0,19$ & $-0,12$ & $-0,19$ & $-0,08$ \\
\hline $32-38 w k s$ & $-0.38 *$ & $-0.47 *$ & $-0,11$ & 0,29 & 0,34 \\
\hline \multicolumn{6}{|l|}{ Diastolic } \\
\hline $10-20 w k s$ & $-0,09$ & 0,1 & 0,03 & 0,01 & $-0,03$ \\
\hline $22-32 w k s$ & $-0,04$ & $-0,11$ & 0,28 & $-0,02$ & 0,23 \\
\hline $32-38 w k s$ & $-0,29$ & 0,15 & $-0,24$ & $0.50^{*}$ & 0,12 \\
\hline \multicolumn{6}{|c|}{ Hemoglobing/dL } \\
\hline $10-20 w k s$ & $-0,31$ & 0,17 & 0,14 & 0,23 & 0,1 \\
\hline $22-32 w k s$ & $-0,17$ & $-0,17$ & 0,06 & 0,11 & $-0,41$ \\
\hline $32-38 w k s$ & $-0,01$ & 0,04 & $-0,21$ & $-0,16$ & $0.02 *$ \\
\hline
\end{tabular}

${ }^{*} \mathbf{p}<0.05$ was considered statistically significant

\section{Discussion}

This study demonstrates fluctuating angiogenic and antiangiogenic profiles (PlGF, sFlt-1, sEng, ratios sFlt1:PlGF and sFlt-1+sEng/PlGF) at specific gestational intervals in a selected group of normotensive pregnantwomen. The antiangiogenic ratio sFlt- $1 / \mathrm{PlGF}$ ratio is suggestive of higher sFlt-1 and lower PlGF levels and is a recognized predictor of HDPs such as PE36. Due to the growing association between circulating angiogenic and anti-angiogenic factors in HDPs, it is necessary to estimate their expressions throughout gestation and their link with risk factors even in normotensive pregnancies. To our knowledge, this is the first South African study to document the angiogenic concentrations at different stages of pregnancy. We previously reported higher se- rum levels of the anti-angiogenic sFlt-1 and soluble endoglin in PE pregnancies compared with normotensive pregnancies irrespective of HIV status, however these were cross-sectional studies conducted at term prior to delivery ${ }^{33,34}$.

Our current study investigated the combination of maternal risk factors and angiogenic profiles throughout the defined gestational intervals and intended to document the concentrations of angiogenic profiles in normotensive pregnancies, in a population that is prone to a high HIV antenatal prevalence. It is hoped that the concentrations documented in our study will assist as a reference range against which these angiogenic and anti-angiogenic factors may be compared in HIV complicated pregnancies. 
Our data shows that changes in diastolic blood pressure, was positively correlated with the maternal anti-angiogenic ratio (sFlt-1/PlGF) at 32-38 weeks' gestation (Table 5). The mean systolic blood pressure measurements were inversely correlated with PlGF and sFlt-1levels at 32-38 weeks' gestation. Similar angiogenic data was previously reported among normotensive pregnancies ${ }^{26,36}$. Previous studies report an increased risk for PE development in healthy nulliparous women in response to elevated systolic and diastolic blood pressures ${ }^{28,37}$. However, systolic and diastolic pressures measured at 13-21 weeks' gestation in pre-eclamptic women with high levels of sFlt-1 was reported to be similar to those in normal, uncomplicated pregnancies $^{36}$. This is suggestive of a healthy vasculature amongst a selected group of participants who were diagnosed with PE, indicative that PE development within this group occured in response to either increased circulating sFlt-1 levels or increased susceptibility to vascular disorders. Thus, it is possible that even slight increases in blood pressure during early to mid gestation may result in vascular alterations. Variations in sFlt-1 during pregnancy in low risk women may be valuable for PE identification in those who lack the conventional risk factors.

Our results which demonstrate an increase PlGF and sFlt1 around 32-38 weeks' gestation (Table 2) concur with other longitudinal studies ${ }^{19,28,38}$. However, the increase appeared earlier in our study, at weeks 22-30 which concurred with Palm and co-workers ${ }^{39}$, in contrast to other studies $^{28,38}$. The rising levels might be caused by a relative placental ischemia as a result of increasing myometrial tone and uterine contractions at the end of pregnancy in preparation for delivery ${ }^{40}$. Moreover, it is possible that the modest expression of PlGF during the 10-20 weeks' gestational interval in our study may be attributed to the preliminary vasculogenic phases and thereafter progressively increases after 22 weeks' gestation due to the angiogenc switch from branching to nonbranching angiogenesis ${ }^{13}$. The rise in serum sFlt-1 levels observed in our study as gestation progresses, is consistent with several studies $^{19,39,41}$. It is likely that this elevation is due to increasing placental ischemia and oxidative stress occurring during pregnancy, indicative of angiogenic restriction, and controlled vascular permeability ${ }^{42}$, and is corroborated by others ${ }^{39,43,44}$.

Our data also suggests a correlation of maternal age with the anti-angiogenic ratio at 32-38 weeks' gestation, which is consistent with Staff and co-workers ${ }^{27}$. However, our data shows no link between these angiogenic proteins with birth weight or hemoglobin levels, indicative that the circulating angiogenic alterations may have little or no effect on neonatal wellbeing, or that our sample size was too small. Nevertheless, our data has shown that hemaglobin levels were lower in the HIV+ve groups in comparison to the HIV-ve groups, despite the lack of statistical significance. In our study, the mean $\mathrm{Hb}$ level was 11.78 and $10.63 \mathrm{~g} / \mathrm{dL}$ in HIV negative and positive women respectively. Anaemia in pregnancy is defined when the hemoglobin level in peripheral blood is $<11 \mathrm{~g} / \mathrm{dL}^{45}$, classified into mild anemia (10-10.09 g/dL), moderate anaemia $(7-9.9 \mathrm{~g} / \mathrm{dL})$ and severe anaemia $(<7 \mathrm{~g} / \mathrm{dL})^{46,47}$. Increased haemoglobin levels is shown to be correlated with elevated body mass index, primiparity, an increased risk of gestational hypertensive disorders and adverse birth outcomes such as low birth weight from third trimester onwards ${ }^{48}$. In our study, we also identifed lower hemaglobin levels in the HIV positive group, suggestive of mild anemia (Table 4). Recent provision of HAART to pregnant women, irrespective of their CD4 counts, has contributed significantly to the reduction in maternal deaths ${ }^{4}$. While HAART has the potential to lower the prevalence and severity of anaemia, mitochondrial toxicity and irregular reticulocyte counts appear to be common effects of zidovudine, which may exacerbate the risk of anemias ${ }^{4}$. A South African report published in 2014 indicate no difference in anemia prevalence in those on HAART in comparison to those who received zidovudine alone during pregnancy ${ }^{49}$, but this warrants further investigation.

A reduction in serum levels of sFlt-1and sEng in nulliparous women between 22-30 week's gestation, and an elevation from 32 weeks onwards was also noted in our study. Despite the lack of statistical significance, a reduction in serum sEng levels at 22-30 weeks' gestation and an elevation at 32 weeks' was observed (Table 2). Nulliparous pregnancies are characterized by higher ischemic placental environments since their spiral arteries have not been formerly remodeled and is therefore identified as a risk factor for PE development ${ }^{40,50}$. It is still unclear why angiogenic/anti-angiogenic profiles are higher in nulliparous women. It is possible that the increased hypoxic environment increases placental sFlt-1 production ${ }^{40}$. The high anti-angiogenic state observed in our study during 
the $3^{\text {rd }}$ gestational interval (32-38 weeks) is consistent with that reported by Bdolah and co workers ${ }^{51}$. The raised and modified angiogenic profile observed in nulliparous pregnant women may be a possible molecular tool that rationalizes the epidemiological association between PE and nulliparity. Sovio and co-workers also pooled the sFlt-1:PlGF ratio at 36 weeks' gestation, with maternal characteristics, and corroborated its clinical value in extrapolating the risk of PE development at term in unselected nulliparous women ${ }^{52}$.

$\mathrm{BMI}$ is also believed to be correlated with angiogenic balance even in uncomplicated pregnancies ${ }^{26,27}$. In our study, the sFlt-1 levels decreased consistently throughout pregnancy amongst those with BMIs $>25 \mathrm{~kg} / \mathrm{m}^{2}$, however, a significant negative correlation was observed at 10-20 weeks. A similar relationship was observed by Mijal and co-workers between second trimester circulating sFlt-1 levels with BMI in normotensive pregnancies ${ }^{20}$. Slight inconsistencies may prevail, since we pooled our $2^{\text {nd }}$ trimester data into a gestational interval at 10-20 weeks, due to late antenatal attendance. Studies comparing BMI at the first antenatal consult with first and second trimester sFlt-1 levels and sFlt-1/PlGF ratio, revealed a positive correlation $^{53}$.

\section{Conclusion}

Since circulating concentrations of angiogenic and anti-angiogenic factors change with gestational age as shown in our study and various others ${ }^{19,26,27,54}$, their inclusion at different gestation levels may expand its clinical value. However, due to the absence of pre-eclampsia cases in our study, our reference values for these angiogenic profiles are not intended for diagnostic use. The angiogenic concentrations described in our study may be used to explore the role of these angiogenic profiles in other placental-related disorders. Our data suggest that the angiogenic profiles during pregnancy may be differentially adjusted based on the varying physiological maternal demands. Our study was limited by a small sample size, which reduced statistical power to explore associations of clinical interest. These results should be confirmed in larger studies.

\section{Acknowledgements}

The authors thank the research administrators for coordination of the project. We also thank the nursing staff at the PHC facility for assistance with participant recruitment and data collection. We are grateful to all the patients who participated in the study.

\section{Conflict of interest}

The authors have no conflicts of interest to disclose.

\section{Author contributions}

All the authors contributed to the study, read and approved the final version, and consent to publication of this manuscript. MO: Laboratory analyses, data capture and management; PR: conception, design, statistical analyses and critical review of manuscript; MNS: conception and design of main study; LOC: conception and design of main study; DB: conception and design of main study; FH: conception and design of main study; SG: conception and design of main study; TN: conception and design of main study; NG: conception, design, analysis of data and writing of manuscript.

\section{Funding}

The research was funded by the SA Medical Research Council (Grant No. DUT/MH1).

\section{References}

1. World Health Organization, UNICEF. Trends in maternal mortality: 1990-2015: estimates from WHO, UNICEF, UNFPA, World Bank Group and the United Nations Population Division.

2. Gumede S, Black V, Naidoo N, Chersich MF. Attendance at antenatal clinics in inner-city Johannesburg, South Africa and its associations with birth outcomes: analysis of data from birth registers at three facilities. BMC Public Health. 2017;17(3):443.

3. Govender T, Reddy P, Ghuman S. Obstetric outcomes and antenatal access among adolescent pregnancies in KwaZulu-Natal, South Africa. S Afr Fam Pract. 2017;1(1):1-7.

4. Sebitloane HM, Moodley D. The impact of highly active antiretroviral therapy on obstetric conditions: A review. Eur J Obstet Gynecol Reprod Biol. 2017;210:126-131.

5. Ramjee G, Wand H, Whitaker C, McCormack S, Padian N, Kelly C, et al. HIV Incidence Among Non-Pregnant Women Living in Selected Rural, Semi-Rural and Urban Areas in Kwazulu-Natal, South Africa,. AIDS Behav. 2012;16:2062-2071. 
6. Pattinson R, Fawcus S, Moodley J. National Committee for Confidential Enquiries into Maternal Deaths. Tenth interim report on Confidential Enquiries into Maternal Deaths in South A frica. 2011 and 2012. 2013.

7. Newell M, Bunders M. Safety of antiretroviral drugs in pregnancy and breastfeeding for mother and child. Curr Opin HIV AIDS. 2013;8:504-510.

8. Wimalasundera R, Larbalestier N, Smith J, de Ruiter A, McGThom S, Hughes A, et al. Pre-eclampsia, antiretroviral therapy, and immune reconstitution. Lancet. 2002;360(9340):1152-1154.

9. Suy A, Martínez E, Coll O, Lonca M, Palacio M, de Lazzari E, et al. Increased risk of pre-eclampsia and fetal death in HIV-infected pregnant women receiving highly active antiretroviral therapy. AIDS. 2006;20(1):59-66.

10. Kalk E, Schubert P, Bettinger JA, Cotton MF, Esser M, Slogrove A, et al. Placental pathology in HIV infection at term: a comparison with HIV-uninfected women. Trop Med Int Health. 2017;22(5):604-613.

11. Kim YW, West XZ, Byzova TV. Inflammation and oxidative stress in angiogenesis and vascular disease. Journal of Molecular Medicine. 2013;91(3):323-328.

12. Ong S, Lash G, Baker PN. Angiogenesis and placental growth in normal and compromised pregnancies. Baillieres Best Pract Res Clin Obstet Gynaecol. 2000;14(6):969-980.

13. Andraweera P, Dekker G, Roberts C. The vascular endothelial growth factor family in adverse pregnancy outcomes. Human Reprod Update. 2012;18(4):436-457.

14. Espinoza J. Angiogenic imbalances in the pathogenesis of pregnancy complications. Fetal Matern Med Rev. 2014;25(1):42-58.

15. Clark DE, Smith SK, He Y, Day KA, Licence DR, Corps AN, et al. A vascular endothelialgrowth factor antagonist is produced by the human placenta and released into the maternal circulation. Biol Reprod. 1998;59(6):15401548.

16. Romero R, Chaiworapongsa T, Erez O, Tarca AL, Gervasi MT, Kusanovic JP, et al. An imbalance between angiogenic and anti-angiogenic factors precedes fetal death in a subset of patients: results of a longitudinal study. J Matern Fetal Neonatal Med. 2010;23(12):1384-1399. 17. Venkatesha S, Toporsian M, Lam C, Hanai J, Mammoto T, Kim YM, et al. Soluble endoglin contributes to the pathogenesis of preeclampsia. Nat Med. 2006;12:642649.

18. Walshe T, Dole V, Maharaj A, Patten I, Wagner D,
D'Amore P. Inhibition of VEGF orTGF- $\{$ beta $\}$ signaling activates endothelium and increases leukocyte rolling. Arteroscler Thromb Vasc Biol. 2009;29:1185-1192.

19. Romero R, Nien JK, Espinoza J, Todem D, Fu W, Chung $\mathrm{H}$, et al. A longitudinal study of angiogenic (placental growth factor) and anti-angiogenic (soluble endoglin and soluble vascular endothelial growth factor receptor-1) factors in normal pregnancy and patients destined to develop preeclampsia and deliver a small for gestational age neonate. J Matern Fetal Neonatal Med. 2008;21(1):9-23. 20. Mijal RS, Holzman CB, Rana S, Karumanchi SA, Wang J, Sikorskii A. Midpregnancy levels of angiogenic markers in relation to maternal characteristics. $A m \mathrm{~J} \mathrm{Ob}$ stet Gynecol. 2012;204(3):244. e241-244. e212.

21. Verlohren S, Perschel FH, Thilaganathan B, Droge LA, Henrich W, Busjahn A, et al. Angiogenic Markers and Cardiovascular Indices in the Prediction of Hypertensive Disorders of Pregnancy. Hypertension. 2017;69(6):11921197.

22. Matsumoto T, Uemura S, Takeda Y, Matsui M, Okada $\mathrm{S}$, Nishida T, et al. An elevated ratio of placental growth factor to soluble fms-like tyrosine kinase-1 predicts adverse outcomes in patients with stable coronary artery disease. Intern Med. 2013;52(10):1019-1027.

23. Fisher $\mathrm{S}$. Why is placentation abnormal in preeclampsia? . Am J Obstet Gynecol. 2015;213:S115-122.

24. Roberts JM, Taylor RN, Musci TJ, Rodgers GM, Hubel CA, McLaughlin MK. Preeclampsia: An endothelial cell disorder. Am J Obstet Gynecol. 1990;32(3):299-299. 25. Jim B, Karumanchi SA. Preeclampsia: Pathogenesis, Prevention, and Long-Term Complications. Semin Nephrol. 2017;37(4):386-397.

26. Troisi R, Braekke K, Harsem NK, Hyer M, Hoover $\mathrm{RN}$, Staff AC. Blood pressure augmentation and maternal circulating concentrations of angiogenic factors at delivery in preeclamptic and uncomplicated pregnancies. Am J Obstet Gynecol. 2008;199(6):653. e651-653. e610.

27. Staff AC, Harsem NK, Braekke K, Hyer M, Hoover RN, Troisi R. Maternal, gestational and neonatal characteristics and maternal angiogenic factors in normotensive pregnancies. Eur J Obstet Gynecol Reprod Biol. 2009;143(1):29-33.

28. Levine RJ, Maynard SE, Qian C, Lim K-H, England LJ, Yu KF, et al. Circulating angiogenic factors and the risk of preeclampsia. N Engl J Med. 2004;350(7):672-683. 29. Coolman M, Timmermans S, de Groot CJ, Russcher 
H, Lindemans J, Hofman A, et al. Angiogenic and fibrinolytic factors in blood during the first half of pregnancy and adverse pregnancy outcomes. Obstetrics \& Gynecology. 2012;119(6):1190-1200.

30. Maynard SE, Karumanchi SA, editors. Angiogenic factors and preeclampsia. Seminars in nephrology; 2011: Elsevier.

31. Verlohren S, Herraiz I, Lapaire O, Schlembach D, Moertl M, Zeisler H, et al. The sFlt-1/PlGF ratio in different types of hypertensive pregnancy disorders and its prognostic potential in preeclamptic patients. American Journal of Obstetrics and Gynecology. 2012;206(1):58. e51-58. e58.

32. Rana S, Powe CE, Salahuddin S, Verlohren S, Perschel $\mathrm{FH}$, Levine RJ, et al. Angiogenic factors and the risk of adverse outcomes in women with suspected preeclampsia. Circulation. 2012: CIRCULATIONAHA. 111.054361. 33. Govender N, Naicker T, Moodley J. Maternal imbalance between pro-angiogenic and anti-angiogenic factors in HIV-infected women with pre-eclampsia. Cardiovasc J Afr. 2013;24(5).

34. Govender N, Naicker T, Rajakumar R, Moodley J. Soluble fms-like tyrosine kinase-1 and soluble endoglin in HIV-associated preeclampsia. Eur J Obstet Gynecol Reprod Biol. 2013;170:100-105.

35. Skinner D.Qualitative methodology: an introduction. 2nd edition ed. Joubert G, Ehrlich R, editors. Cape Town: Oxford University Press Southern Africa; 2007.

36. Levine RJ, Qian C, Maynard SE, Kai FY, Epstein FH, Karumanchi SA. Serum sFlt1 concentration during preeclampsia and mid trimester blood pressure in healthy nulliparous women. Am J Obstet Gynecol. 2006;194(4):10341041.

37. Sibai BM, Ewell M, Levine RJ, Klebanoff MA, Esterlitz J, Catalano PM, et al. Risk factors associated with preeclampsia in healthy nulliparous women. Am J Obstet Gynecol. 1997;177(5):1003-1010.

38. Chaiworapongsa T, Romero R, Kim YM, Kim GJ, Kim MR, Espinoza J, et al. Plasma soluble vascular endothelial growth factor receptor-1 concentration is elevated prior to the clinical diagnosis of pre-eclampsia. J Matern Fetal Neonatal Med. 2005;17(1):3-18.

39. Palm M, Basu S, Larsson A, Wernroth L, Åkerud H, Axelsson O. A longitudinal study of plasma levels of soluble fms-like tyrosine kinase 1 (sFlt1), placental growth factor (PlGF), sFlt1: PlGF ratio and vascular endothelial growth factor (VEGF-A) in normal pregnancy. Acta Obstet Gynecol Scand. 2011;90(11):1244-1251.

40. Karumanchi SA, Bdolah Y. Hypoxia and sFlt-1 in preeclampsia: the "chicken-and-egg" question. Endocrinology. 2004;145(11):4835-4837.

41. Sundrani D, Khot V, Pisal H, Mehendale S, Wagh G, Joshi A, et al. Gestation dependant changes in angiogenic factors and their associations with fetal growth measures in normotensive pregnancy. PLoS One. 2013;8(1):e54153. 42. Ni Y, May V, Braas K, Osol G. Pregnancy augments uteroplacental vascular endothelial growth factor gene expression and vasodilator effects. Am J Physiol Heart Circ Physiol. 1997;273(2):H938-H944.

43. Redman CWG, Sargent IL. Placental stress and pre-eclampsia: a revised view. Placenta. 2009;30:38-42.

44. Ozkan Y, Yardim-Akaydin S, Erdem A, Şimşek B. Variability of total thiol compounds, oxidative and nitrosative stress in uncomplicated pregnant women and nonpregnant women. Arch Gynecol Obstet. 2012;285(5):13191324.

45. Buseri F,Uko E, Jeremiah Z, Usanga E. Prevalence and risk factors of anaemia among pregnant women in Nigeria. Open Hematol J. 2008;2(1).

46. Grewal A. Anaemia and pregnancy: Anaesthetic implications. Indian J Anaesth. 2010;54(5):380.

47. Regil LMD, Penarosas JP, Cusick S, Lynch S. Haemoglobin concentration for the diagnosis of anemia and assessment of severity. Vitamin and mineral nutrition information system, department of nutrition for health and development. WHO. 2011.

48. Gaillard R, Eilers PHC, Yassine S, Hofman A, Steegers EAP, Jaddoe VWV. Risk Factors and Consequences of Maternal Anaemia and Elevated Haemoglobin Levels during Pregnancy: a Population-Based Prospective Cohort Study. Paediatr Perinat Epidemiol. 2014;28: 213-226. 49. Nandlal V, Moodley D, Grobler A, Bagratee J, Maharaj NR, Richardson P. Anaemia in pregnancy is associated with advanced HIV disease. PloS one. 2014;9(9):e106103. 50. Duckitt K, Harrington D. Risk factors for pre-eclampsia at antenatal booking: systematic review of controlled studies. BMJ. 2005;330(7491):565.

51. Bdolah Y, Elchalal U, Natanson-Yaron S, Yechiam H, Bdolah-Abram T, Greenfield C, et al. Relationship between nulliparity and preeclampsia may be explained by altered circulating soluble fms-like tyrosine kinase 1. Hypertens Pregnancy. 2014;33(2):250-259. 
52. Sovio U, Gaccioli F, Cook E, Hund M, Charnock-Jones DS, Smith GCS. Prediction of Preeclampsia Using the Soluble fms-Like Tyrosine Kinase 1 to Placental Growth Factor Ratio. Hypertension. 2017: HYPERTENSIONAHA. 116.08620.

53. Faupel-Badger JM, Staff AC, Thadhani R, Powe CE, Potischman N, Hoover RN, et al. Maternal angiogenic profile in pregnancies that remain normotensive. Eur J Obstet Gynecol Reprod Biol. 2011;158(2):189-193.

54. Wataganara T, Pratumvinit B, Lahfahroengron P, Pooliam J, Talungchit P, Leetheeragul J, et al. Circulating soluble fms-like tyrosine kinase-1 and placental growth factor from 10 to 40 weeks' pregnancy in normotensive women. Journal of Perinat Med. 2017:1-7. 